\title{
THE FIRST HITTING DISTRIBUTION OF A SPHERE FOR SYMMETRIC STABLE PROCESSES
}

\author{
BY \\ SIDNEY C. PORT
}

1. Introduction. Let $X(t)$ be a symmetric stable process on $N$ dimensional Euclidean space $R^{N}$, having exponent $\alpha$ and transition density

$$
p(t, x)=(2 \pi)^{-N} \int_{R^{N}} \exp \left[-t|\theta|^{\alpha}\right] e^{-i(\theta, x)} d \theta .
$$

We will always work with the version of the process $X(t)$ which is a standard Markov process. (See Chapter 1 of [1] for a complete description of a standard process.) For any $r>0$ let $S_{r}=\left\{y \in R^{N}:|y|=r\right\}$ denote the sphere of center 0 and radius $r$. Set $T_{r}=\inf \{t>0:|X(t)|=r\}$, and, as usual, set $T_{r}=\infty$ if $|X(t)| \neq r$ for all $t>0$. The hitting measure and Green's function of $S_{r}$ are respectively the quantities,

$$
H_{r}(x, d \xi)=P_{x}\left(X\left(T_{r}\right) \in d \xi, T_{r}<\infty\right)
$$

and $g_{r}(x, y)$, where $g_{r}(x, y)$ is the density of the measure

$$
\int_{0}^{\infty} P_{x}\left(T_{r}>t, X(t) \in d y\right) d t
$$

The hitting probability of $S_{r}$ is $\Phi_{r}(x)=P_{x}\left(T_{r}<\infty\right)$. Our purpose in this paper is to explicitly compute these as well as some related quantities.

In brief we will do the following. In $\$ 2$ we introduce the radial process $Z_{\alpha}(t)$ and use it to compute $\Phi_{r}(x)$ by the relation $\Phi_{r}(x)=P_{|x|}\left(\tau_{r}<\infty\right)$, where $\tau_{r}=$ $\inf \left\{t>0: Z_{\alpha}(t)=r\right\}$. The problem is trivial if $\alpha \leqq 1$ (see Proposition 2.1) since $\{r\}$ is a polar set for $Z_{\alpha}(t)$ in that case. Also, if the process is recurrent, then $\Phi_{r}(x) \equiv 1$ so the only cases of interest are $1<\alpha<N$. Our technique here is simply to note that

$$
\Phi_{r}(x)=\frac{u(x, r)}{u(r, r)}
$$

where $u(x, r)$ is the potential kernel of $Z_{\alpha}(t)$, and to compute $u(x, y)$. But having $u(x, y)$ one may explicitly compute more elaborate probabilities, e.g.,

$$
P_{x}\left(\operatorname{Min}_{1 \leqq i \leqq n} T_{r_{t}}=T_{r_{j}}\right) .
$$

Some of these computations will be also carried out in $\$ 2$. In $\S 3$ we compute $H_{r}(x, d \xi)$ by the method devised by M. Riesz [8] of inversion in an appropriate sphere, and in $\S 4$ we use the results of $\S 2$ and 3 to write down the Green's function of $S_{r}$.

Received by the editors August 26, 1967. 
Previously, the above quantities were computed for the solid ball by Blumenthal, Getoor, and Ray in [3] by the use of Riesz's inversion technique, and in [7] the author computed these quantities for arbitrary finite sets in the case of recurrent one-dimensional stable processes with exponent $\alpha>1$.

2. The radial process. Since $X(t)$ is isotropic, the process $Z_{\alpha}(t)=|X(t)|$ is a Markov process, and since $X(t)$ is a Feller process, it must be that $Z_{\alpha}(t)$ is also a Feller process. Thus by $\S 9$ of [1], there is a realization of $Z_{\alpha}(t)$ as a standard Markov process. We henceforth assume that $Z_{\alpha}(t)$ is this version of the process. If $\alpha=2$, then $X(t)$ is Brownian motion, and it is well known (see [6], p. 60 or [2], §4) that the transition function of $Z_{2}(t)$ is given by

$$
P_{x}\left(Z_{2}(t) \in A\right)=\int_{A} f_{2}(t, x, y) \mu(d y)
$$

where $\mu(d y)=2^{-N / 2}[\Gamma((N / 2)+1)]^{-1} y^{N} d y$, and

$$
f_{2}(t, x, y)=\Gamma\left(\frac{N}{2}\right)(2 t)^{-1}\left(\frac{x y}{2}\right)^{1-N / 2} \exp \left[-\left(x^{2}+y^{2}\right) / 4 t\right] I_{N / 2-1}\left(\frac{x y}{2 t}\right),
$$

where $I_{v}$ is the usual modified Bessel function.

Let $T_{\beta}$ be the stable subordinator of exponent $\beta, 0<\beta<1$, with $T_{\beta}(0)=0$. Then it is a familiar fact that $Z_{\alpha}$ and $Z_{2}\left(T_{\alpha / 2}(t)\right)$ are equivalent provided that $T_{\alpha / 2}$ and $Z_{2}$ are independent. Let $h_{\alpha / 2}(t, u)$ denote the density function of $T_{\alpha / 2}$. Then the transition function of $Z_{\alpha}$ is given by

$$
P_{x}\left(Z_{\alpha}(t) \in A\right)=\int_{A} f_{\alpha}(t, x, y) \mu(d y)
$$

where $\mu$ was defined above and

$$
f_{\alpha}(t, x, y)=\int_{0}^{\infty} h_{\alpha / 2}(t, u) f_{2}(u, x, y) d u .
$$

Let $\tau_{r}=\inf \left\{t>0: Z_{\alpha}(t)=r\right\} \quad\left(=\infty\right.$ if $Z_{\alpha}(t) \neq r$ for all $\left.t>0\right)$. It is clear that $\{r\}$ is a polar set for the radial process $Z_{\alpha}(t)$ if and only if the sphere $S_{r}=\{y:|y|=r\}$ is a polar set for the process $X(t)$. In addition, if $r$ is a regular point of $\{r\}$ for the radial process, then all points on the sphere $S_{r}$ are regular for this sphere for the process $X(t)$. The following fact ensues from Corollary 4.3 and Theorem 3.1 of [2]. For completeness, we sketch below an alternate proof which avoids the use of Hunt's capacity theory.

Proposition 2.1. For the radial process $Z_{\alpha}(t), r$ is regular for $\{r\}$ provided $\alpha>1$. If $\alpha \leqq 1$, then $\{r\}$ is polar.

Proof. Let $A_{n}=\left\{x \in R^{1}:|x-r|<1 / n\right\}$ and let $\tau_{n}$ be the first hitting time of $A_{n}$. Set

$$
H_{A_{n}}^{\lambda}(x, B)=E_{x}\left(\exp \left(-\lambda \tau_{n}\right) 1_{B}\left(x\left(\tau_{n}\right)\right) ; \tau_{n}<\infty\right)
$$


and let $u^{\lambda}(x, y)$ be the Laplace transform of $f_{\alpha}(t, x, y)$. Then the usual first passage arguments show that

$$
u^{\lambda}(x, r)=\int_{\bar{A}_{n}} H_{A_{n}}^{\lambda}(x, d z) u^{\lambda}(z, r)
$$

Simple computations (see the proof of Corollary 4.3 of [2] for details) show that if $r>0$, then

$$
f_{\alpha}(t, r, r) \sim k t^{-1 / \alpha}, \quad t \rightarrow 0,
$$

where $k$ is some constant (dependent on $r$ ) $>0$. Thus $u^{\lambda}(x, r) \rightarrow \infty$ as $x \rightarrow r$ when $\alpha \leqq 1$, while for $\alpha>1, u^{\lambda}(x, r)$ is bounded and continuous in $x$ in a neighborhood of $r$.

Suppose $\alpha \leqq 1$. Then (2.3) shows that

$$
\infty>u^{\lambda}(x, r) \geqq E_{x}\left(\exp \left(-\lambda \tau_{r}\right) ; \tau_{r}<\infty\right) \inf _{z \in A_{n}} u^{\lambda}(z, r),
$$

and it follows that $P_{x}\left(\tau_{r}<\infty\right)=0$ for all $x \neq r$. Since

$$
P_{r}\left(\tau_{r}<\infty\right)=\lim _{t \downarrow 0} \int_{R^{N}} f_{\alpha}(t, r, y) P_{y}\left(\tau_{r}<\infty\right) d y
$$

we see that $\{r\}$ is polar for all $r$.

Now suppose $\alpha>1$. Since $Z_{\alpha}(t)$ is a standard process it is quasi-left continuous (see [1], $\S 9$ for a definition) and thus $\tau_{n} \uparrow \tau_{r}$ and $X\left(\tau_{n}\right) \rightarrow X\left(\tau_{r}\right)$, a.s. $P_{x}, x \neq r$. It then follows from (2.3) that for $x \neq r$ and $r>0$,

$$
u^{\lambda}(x, r) / u^{\lambda}(r, r)=E_{x}\left(\exp \left(-\lambda \tau_{r}\right), \tau_{r}<\infty\right)
$$

Hence

$$
\lim _{x \rightarrow r} E_{x}\left(\exp \left(-\lambda \tau_{r}\right), \tau_{r}<\infty\right)=1,
$$

and it follows easily from this that $r$ is regular for $\{r\}$ whenever $r>0$. This completes the proof.

In view of the above result we shall henceforth only consider the processes with $\alpha>1$. If the processes are recurrent, then $\Phi_{r}(x) \equiv 1$, so we need only consider transient processes (i.e., $\alpha<N$ ).

TheORem 2.1. Assume $1<\alpha<N$. Then for $r>0$,

$$
\Phi_{r}(x)=\left.\frac{\pi^{1 / 2} \Gamma\left(\frac{\alpha+N}{2}-1\right) 2^{2-\alpha}}{\Gamma\left(\frac{\alpha-1}{2}\right)} r^{N-\alpha}|| x\right|^{2}-\left.r^{2}\right|^{(\alpha / 2)-1} P_{-\alpha / 2}^{1-N / 2}\left(\frac{|x|^{2}+r^{2}}{\left.|| x\right|^{2}-r^{2} \mid}\right)(|x| r)^{1-N / 2},
$$

where $P_{\mu}^{v}$ is the usual Legendre function of the first kind. 
Proof. Since the processes are transient, $u^{\lambda}(x, y) \rightarrow u(x, y), \lambda \downarrow 0$, where $u(x, y)$ is the potential kernel of $Z_{\alpha}(t)$. From (2.4) we see that

$$
P_{x}\left(\tau_{r}<\infty\right)=u(x, r) / u(r, r),
$$

so to establish (2.6) we need to compute the right-hand side of (2.7). This will be done in the following two lemmas.

LEMMA 2.1. If $1<\alpha<N$, then

$$
u_{\alpha}(x, y)=\frac{\Gamma\left(\frac{N}{2}\right) \Gamma\left(\frac{N-\alpha}{2}\right) 2^{(N / 2)-\alpha}}{\Gamma(\alpha / 2)}(x y)^{1-N / 2}\left|x^{2}-y^{2} \cdot\right|^{(\alpha / 2)-1} P_{-\alpha / 2}^{1-N / 2}\left(\frac{x^{2}+y^{2}}{\left|x^{2}-y^{2}\right|}\right),
$$

where $P_{\mu}^{v}$ is the usual Legendre function of the first kind.

Proof. The stable subordinator $T_{\alpha / 2}$ of exponent $\alpha / 2$ is the unique stable process on $(0, \infty)$ whose transition density has Laplace transform

and thus

$$
\int_{0}^{\infty} h_{\alpha / 2}(t, u) e^{-\gamma u} d u=\exp \left(-t \gamma^{\alpha / 2}\right),
$$

$$
\int_{0}^{\infty} \int_{0}^{\infty} h_{\alpha / 2}(t, u) e^{-\gamma u} d u d t=\gamma^{-\alpha / 2}
$$

Hence the potential kernel of $T_{\alpha / 2}$ is $\Gamma(\alpha / 2)^{-1} u^{(\alpha / 2)-1}$. From (2.2) we then see that

$$
u_{\alpha}(x, y)=\frac{1}{\Gamma(\alpha / 2)} \int_{0}^{\infty} u^{(\alpha / 2)-1} f_{2}(u, x, y) d u .
$$

Using the explicit formula for $f_{2}(u, x, y)$ and formula 8, p. 196 of [4], we obtain (2.8).

LEMMA 2.2. If $1<\alpha<N$, then for $r>0$,

$$
u_{\alpha}(r, r)=\frac{\pi^{-1 / 2} 2^{\alpha-2} \Gamma\left(\frac{\alpha-1}{2}\right)}{\Gamma\left(\frac{\alpha+N}{2}-1\right)} \frac{\Gamma\left(\frac{N}{2}\right) \Gamma\left(\frac{N-\alpha}{2}\right) 2^{(N / 2)-\alpha}}{\Gamma(\alpha / 2)} r^{\alpha-N} .
$$

Proof. This follows from (2.8) and the asymptotic relation (see formula 20, p. 164 of [5]),

$$
P_{-\alpha / 2}^{1-N / 2}\left(\frac{x^{2}+y^{2}}{\left|x^{2}-y^{2}\right|}\right) \sim \frac{\pi^{-1 / 2} 2^{\alpha-2} \Gamma\left(\frac{\alpha-1}{2}\right)}{\Gamma\left(\frac{\alpha+N}{2}-1\right)} y^{\alpha-2}\left|x^{2}-y^{2}\right|^{1-\alpha / 2}, \quad x \rightarrow y .
$$

Corollary 2.1. Assume $1<\alpha<N$. Then for any $r>0$,

$$
\Phi_{r}(0)=\frac{\Gamma\left(\frac{\alpha+N}{2}-1\right) \pi^{1 / 2} 2^{2-\alpha}}{\Gamma(N / 2) \Gamma\left(\frac{\alpha-1}{2}\right)} .
$$


Proof. This follows from (2.6) and the asymptotic formula (see formula 3 of p. 163 of [5]) that for $y>0$,

$$
P_{-\alpha / 2}^{1-N / 2}\left(\frac{x^{2}+y^{2}}{\left|x^{2}-y^{2}\right|}\right) \sim \frac{y^{(1-N / 2)}}{\Gamma(N / 2)} x^{-(1-N / 2)}, \quad x \rightarrow 0 .
$$

COROLlary 2.2. Assume $1<\alpha<N$. Then the capacity of the sphere of radius $r$ is $C_{r}$ where

$$
C_{r}=\frac{4 \Gamma\left(\frac{\alpha+N}{2}-1\right) \pi^{(N+1) / 2} \Gamma(\alpha / 2)}{\Gamma\left(\frac{N}{2}\right) \Gamma\left(\frac{\alpha-1}{2}\right) \Gamma\left(\frac{N-\alpha}{2}\right)} r^{N-\alpha} .
$$

Proof. Let $\pi_{r}(d \xi)$ be the capacitory measure of the sphere $S_{r}=\{x:|x|=r\}$. The potential kernel of $X(t)$ is just the Riesz kernel $K|x|^{\alpha-N}$, where

$$
K=\frac{\Gamma((N-\alpha) / 2)}{4^{\alpha / 2} \pi^{N / 2} \Gamma(\alpha / 2)}
$$

and it is a basic fact that (see Chapter 6 of [1]) the capacitory potential of $S_{r}$ is just $\Phi_{r}(x)$, i.e.,

$$
\Phi_{r}(x)=\int_{S_{r}} K|y-x|^{\alpha-N_{r}} \pi_{r}(d y) .
$$

Thus $\Phi_{r}(0)=r^{\alpha-N} K \pi\left(S_{r}\right)$. Since $C_{r}=\pi\left(S_{r}\right),(2.11)$ follows from (2.10) and the above relation.

For $\alpha=2$ (i.e., Brownian motion) and $N>2$ the formula for $u(x, y)$ is considerably simpler.

$$
u(x, y)=2^{(N / 2)-2} \Gamma(N / 2)(N / 2-1)^{-1}[\operatorname{Max}(x, y)]^{2-N} .
$$

Using this, it is easily seen that Theorem 2.1 yields the well-known result

$$
\begin{aligned}
\Phi_{r}(x) & =1, & & x \leqq r, \\
& =(x / r)^{2-N}, & & x>r .
\end{aligned}
$$

By the same type of arguments we may compute more elaborate hitting probabilities for the processes with $1<\alpha<N$. Let $B=\left\{r_{1}, r_{2}, \ldots, r_{n}\right\}$ where $r_{1}<r_{2}<\cdots$ $<r_{n}$. Since potential $\sum_{i=1}^{n} u\left(x, r_{i}\right) \mu_{i}$ on $N$ uniquely determines the numbers $\mu_{i}$, we see that the matrix $U_{i j}=u\left(r_{i}, r_{j}\right)$ is invertible. Denote its inverse by $K_{B}(i, j)$. If $\tau_{B}$ is the first hitting time of $B$ by the radial process, and if

$$
T_{B}=\inf \{t>0:|X(t)| \in B\}
$$

then, of course, $P_{x}\left(T_{B}<\infty\right)=P_{|x|}\left(\tau_{B}<\infty\right)$. However, it is a fundamental fact in the theory of Markov processes (see [1, Chapter 6]) that there is a bounded measure $\pi$ having support on $B$ such that

$$
P_{a}\left(\tau_{B}<\infty\right)=\sum_{j=1}^{n} u\left(a, r_{j}\right) \pi_{j} .
$$

[This fact may also be proved directly for the $Z_{\alpha}(t)$ process by an argument very similar to that used to deduce (2.7).] Since every point of $B$ is regular for $B$, we 
see that $\pi$ is the unique measure on $B$ such that $1=(U \pi)_{j}, 1 \leqq j \leqq n$, and thus $\pi_{j}=$ $\sum_{i=1}^{n} K_{B}(i, j)$. Consequently

$$
P_{a}\left(\tau_{B}<\infty\right)=\sum_{i=1}^{n} \sum_{j=1}^{n} u\left(a, r_{j}\right) K_{B}(i, j) .
$$

In a similar manner we see that

Set

$$
P_{x}\left(\operatorname{Min}_{1 \leqq i \leqq n}\left(T_{r_{i}}\right)=T_{r_{j}}, T_{B}<\infty\right)=P_{|x|}\left(Z_{\alpha}\left(\tau_{B}\right)=r_{j}, \tau_{B}<\infty\right)
$$

$$
H_{B}\left(a, r_{i}\right)=P_{a}\left(Z_{\alpha}\left(\tau_{B}\right)=r_{i}, \tau_{B}<\infty\right) .
$$

Then the $H_{B}\left(a, r_{i}\right)$ are uniquely determined by the equations

and thus

$$
u\left(a, r_{j}\right)=\sum_{i=1}^{n} H_{B}\left(a, r_{i}\right) u\left(r_{i}, r_{j}\right), \quad 1 \leqq j \leqq n,
$$

$$
H_{B}\left(a, r_{j}\right)=\sum_{i=1}^{n} u\left(a, r_{i}\right) K_{B}\left(r_{i}, r_{j}\right)
$$

For a two point set $B=\left\{r_{1}, r_{2}\right\}$

$$
K_{B}=\frac{1}{\Delta}\left(\begin{array}{rr}
U_{22} & -U_{12} \\
-U_{12} & U_{11}
\end{array}\right)
$$

where $\Delta=U_{11} U_{22}-\left(U_{12}\right)^{2}$. Equations (2.14) and (2.15) then yield

$$
\begin{aligned}
P_{x}\left(T_{B}<\infty\right)= & \frac{u\left(|x|, r_{1}\right) u\left(r_{2}, r_{2}\right)+u\left(|x|, r_{2}\right) u\left(r_{1}, r_{1}\right)}{u\left(r_{1}, r_{1}\right) u\left(r_{2}, r_{2}\right)-u\left(r_{1}, r_{2}\right)^{2}} \\
& -\frac{u\left(r_{1}, r_{2}\right)\left[u\left(|x|, r_{1}\right)+u\left(|x|, r_{2}\right)\right]}{u\left(r_{1}, r_{1}\right) u\left(r_{2}, r_{2}\right)-u\left(r_{1}, r_{2}\right)^{2}}
\end{aligned}
$$

and

$$
\begin{aligned}
& P_{x}\left(T_{r_{1}}<T_{r_{2}}\right)=\frac{u\left(|x|, r_{1}\right) u\left(r_{2}, r_{2}\right)-u\left(|x|, r_{2}\right) u\left(r_{2}, r_{1}\right)}{u\left(r_{1}, r_{1}\right) u\left(r_{2}, r_{2}\right)-u\left(r_{1}, r_{2}\right)^{2}} \\
& P_{x}\left(T_{r_{2}}<T_{r_{1}}\right)=\frac{u\left(|x|, r_{2}\right) u\left(r_{1}, r_{1}\right)-u\left(|x|, r_{1}\right) u\left(r_{1}, r_{2}\right)}{u\left(r_{1}, r_{1}\right) u\left(r_{2}, r_{2}\right)-u\left(r_{1}, r_{2}\right)^{2}}
\end{aligned}
$$

In particular, for $\alpha=2$ we obtain the following well-known results for Brownian motion in dimension $N \geqq 3$.

and

$$
\begin{aligned}
P_{x}\left(T_{r_{1}}<T_{r_{2}}\right) & =1, & & |x| \leqq r_{1}, \\
& =0, & & |x| \geqq r_{2}, \\
& =\frac{|x|^{2-N}-r_{2}^{2-N}}{r_{1}^{2-N}-r_{2}^{2-N}}, & & r_{1} \leqq|x| \leqq r_{2},
\end{aligned}
$$

$$
\begin{aligned}
P_{x}\left(T_{r_{2}}<T_{r_{1}}\right) & =0, & & |x| \leqq r_{1}, \\
& =\left|x / r_{2}\right|^{2-N}, & & |x| \geqq r_{2}, \\
& =\frac{r_{1}^{2-N}-|x|^{2-N}}{r_{1}^{2-N}-r_{2}^{2-N}} . & & r_{1} \leqq|x| \leqq r_{2} .
\end{aligned}
$$


In the above discussion we omitted those processes with $\alpha \geqq N$. We will now fill in this detail. If $N=1$, then since $\alpha>1$, the processes are point recurrent, and the above methods are not directly applicable since $u(x, y)=\infty$. However, in this case a sphere consists of two points, and explicit formulas for the hitting distribution of finite sets were given in [7] (see §3). Alternately, it is easily seen that the recurrent potential kernel of the $Z_{\alpha}(t)$ process is given by $u(x, y)=a(y-x)+a(y+x)$ where $a(x)$ is the recurrent potential kernel of $X(t)$ given in [7]. With this $u$, the hitting distribution is again given by (2.15). The remaining process is $\alpha=N=2$, i.e., planar Brownian motion. Owing to the continuity of the paths, the hitting probabilities for a finite $B$ can be reduced to that of a two point set. But for such a set the result is well known. (See, e.g., [6, p. 62].)

3. The hitting measure of $S_{r}$. Assume $1<\alpha<N$. It is intuitively clear that the capacitory measure $\pi_{r}(d \xi)$ of $S_{r}$ is $C_{r} d \sigma_{r}(\xi)$, where here and in the following, $\sigma_{r}$ is the uniform measure on $S_{r}$, and $C_{r}$ is the capacity of $S_{r}$ given in (2.11). To establish this fact rigorously we note that since every point of $S_{r}$ is regular for $S_{r}$, the measure $\pi_{r}$ is the unique bounded measure having support on $S_{r}$ such that for all $x \in S_{r}$,

$$
1=K \int_{S_{r}}|\xi-x|^{\alpha-N} \pi_{r}(d \xi)
$$

where here and in the following,

$$
K=\frac{\Gamma((N-\alpha) / 2)}{4^{\alpha / 2} \pi^{N / 2} \Gamma(\alpha / 2)}
$$

A change to spherical coordinates now easily shows that $C_{r} d \sigma_{r}(\xi)$ satisfies (3.1). The main result of this section is the following

THEOREM 3.1. Assume $1<\alpha<N$. Then the hitting measure $H_{r}(x, d \xi)$ of $S_{r}$ is given by

$$
H_{r}(x, d \xi)=\left.\frac{\Gamma\left(\frac{\alpha+N}{2}-1\right) \pi^{1 / 2} 2^{2-\alpha} r^{N-\alpha}}{\Gamma(N / 2) \Gamma\left(\frac{\alpha-1}{2}\right)}|| x\right|^{2}-\left.r^{2}\right|^{\alpha-1}|\xi-x|^{2-\alpha-N} d \sigma_{r}(\xi), \quad|x| \neq r,
$$

while $H_{r}(x, d \xi)$ is the unit mass at $x$ if $|x|=r$.

REMARK. The basis of the computation of $H_{r}(x, d \xi)$ which we shall use here is that of inversion in an appropriate sphere orthogonal to $S_{r}$. This idea was first used by M. Riesz in [8] to compute (in probabilistic terminology) the hitting measure of the solid ball. Later, Blumenthal, Getoor, and Ray [3] extended these computations to complete the story for the ball. 
Proof. Consider first the case when $|x|>r$. The inversion in the sphere $\{y:|y-x|=a\}$ is the change of variable $y \rightarrow y^{\prime}=x+a^{2}(y-x)|y-x|^{-2}$. Choose $a^{2}=|x|^{2}-r^{2}$. Then the sphere $S_{r}$ and the inverting sphere are orthogonal, and thus the transformation maps $S_{r}$ onto $S_{r}$. If $y^{\prime}, z^{\prime}$ are the images of $y, z$ under this inversion, then

$$
\left|y^{\prime}-z^{\prime}\right|=\frac{a^{2}|y-z|}{|y-x||z-x|}
$$

Define a measure $\mu_{x}(d \xi)$ on $S_{r}$ by

$$
\mu_{x}(d \xi)|\xi-x|^{\alpha-N}=\pi_{r}\left(d \xi^{\prime}\right)
$$

where $\xi^{\prime}$ is the image of $\xi$, and $\pi_{r}$ is the capacitory measure of $S_{r}$. If $z \in S_{r}$ then so does $z^{\prime}$, and (3.1), (3.4), and (3.5) now show that if $z \in S_{r}$

$$
1=K \int_{S_{r}}\left|z^{\prime}-y^{\prime}\right|^{\alpha-N} \pi_{r}\left(d y^{\prime}\right)=K\left[\frac{a^{2}}{|z-x|}\right]^{\alpha-N} \int_{S_{r}}|z-y|^{\alpha-N} \mu_{x}(d y) .
$$

Thus if $z \in S_{r}$,

$$
K|z-x|^{\alpha-N}=K\left(a^{2}\right)^{\alpha-N} \int_{S_{r}} K|z-y|^{\alpha-N} \mu_{x}(d y) .
$$

But since every point of $S_{r}$ is regular for $S_{r}$, the measure $H_{r}(x, d \xi)$ is the unique measure supported on $S_{r}$ such that

$$
K|z-x|^{\alpha-N}=\int_{S_{r}} H_{r}(x, d \xi) K|z-\xi|^{\alpha-N}, \quad z \in S_{r}
$$

Thus

$$
H_{r}(x, d \xi)=K\left(a^{2}\right)^{\alpha-N} \mu_{x}(d \xi) .
$$

Suppose $\mu_{x}(d \xi)=k_{x}(\xi) d \sigma_{r}(\xi)$. Then (3.5) shows that

$$
k_{x}(\xi)=C_{r}|\xi-x|^{N-\alpha} d \sigma\left(\xi^{\prime}\right) / d \sigma(\xi) .
$$

However, it is clear from the geometry that

$$
\frac{d \sigma\left(\xi^{\prime}\right)}{\left|\xi^{\prime}-x\right|^{N-1}}=\frac{d \sigma(\xi)}{|\xi-x|^{N-1}}
$$

and thus, using (3.4), we find that

$$
k_{x}(\xi)=C_{r}\left(a^{2}\right)^{N-1}|\xi-x|^{2-\alpha-N},
$$

and thus by (3.8),

$$
H_{r}(x, d \xi)=\left.K C_{r}|| x\right|^{2}-\left.r^{2}\right|^{\alpha-1}|\xi-x|^{2-\alpha-N} d \sigma_{r}(\xi), \quad|x|>r .
$$

Suppose now that $|x|<r$. An inversion in the sphere $S_{r}$ sends $x$ to $x^{\prime}=r^{2} x /|x|^{2}$, 
and by what has just been shown above we know that $H_{r}\left(x^{\prime}, d \xi\right)$ satisfies the equation

$$
K\left|y-x^{\prime}\right|^{\alpha-N}=\left.\int_{S_{r}} K^{2} C_{r}|| x^{\prime}\right|^{2}-\left.r^{2}\right|^{\alpha-1}\left|\xi-x^{\prime}\right|^{2-\alpha-N}|\xi-y|^{\alpha-N} d \sigma_{r}(\xi), \quad y \in S_{r} .
$$

But

$$
\left|y-x^{\prime}\right|=r|y-x| /|x|, \quad\left[\left|x^{\prime}\right|^{2}-r^{2}\right]=r^{2}\left[r^{2}-|x|^{2}\right] /|x|^{2},
$$

and thus we find that

$$
H_{r}(x, d \xi)=\left.K C_{r}|| x\right|^{2}-\left.r^{2}\right|^{\alpha-1}|\xi-x|^{2-\alpha-N} d \sigma_{r}(\xi)
$$

satisfies (3.7). This completes the proof.

We note that for $\alpha=2$, i.e., Brownian motion, the kernel in (3.3) becomes the classical Poisson kernel (as it should), and that for a general $\alpha$, the kernel is a very close analogue of this classical kernel.

We conclude this section with a comment on the quantity $H_{r}(x, d \xi)$ in the case of a recurrent stable process, $\alpha \geqq N$. For $\alpha=N=2$, i.e., planar Brownian motion, it is well known that (3.3) still gives the correct result. For $\alpha>1=N$, the sphere is a two point set, and an explicit formula for $H_{r}(x, d \xi)$ was computed in [7, §3].

4. The Green's function. Again we consider the case when $1<\alpha<N$. The Green's function $g_{r}(x, y)$ for the sphere $S_{r}=\{y:|y|=r\}$ is uniquely defined by

$$
g_{r}(x, y) \equiv K|y-x|^{\alpha-N}-\left.K^{2} C_{r} \int_{S_{r}}|| x\right|^{2}-\left.r^{2}\right|^{\alpha-1}|\xi-x|^{2-\alpha-N}|\xi-y|^{\alpha-N} d \sigma_{r}(\xi)
$$

where $K$ is given in (3.2) and $C_{r}$ is the capacity given in (2.11). Set

$$
I=\int_{S_{r}}|\xi-x|^{2-\alpha-N}|\xi-y|^{\alpha-N} d \sigma_{r}(\xi) .
$$

Consider the case when $|x|>r$. An inversion in the sphere $\left\{y:|y-x|^{2}=|x|^{2}-r^{2}\right\}$ sends $\xi \rightarrow \xi^{\prime} \in S_{r}$ and $y \rightarrow y^{\prime}$. Performing this change of variable we find that

$$
\begin{aligned}
I & =\left(|x|^{2}-r^{2}\right)^{1-N}\left|y^{\prime}-x\right|^{N-\alpha} \int_{S_{r}}\left|\xi^{\prime}-y^{\prime}\right|^{\alpha-N} d \sigma_{r}\left(\xi^{\prime}\right) \\
& =\left(|x|^{2}-r^{2}\right)^{1-N}\left|y^{\prime}-x\right|^{N-\alpha} \Phi_{r}\left(y^{\prime}\right)\left(K C_{r}\right)^{-1} \\
& =\left(K C_{r}\right)^{-1}\left(|x|^{2}-r^{2}\right)^{1-\alpha}|y-x|^{\alpha-N} \Phi_{r}\left(y^{\prime}\right) .
\end{aligned}
$$

Substituting this expression for $I$ into (4.1) shows that

$$
g_{r}(x, y)=K|y-x|^{\alpha-N}\left[1-\Phi_{r}\left(y^{\prime}\right)\right], \quad|x|>r .
$$

Now a simple computation shows that

$$
\left|y^{\prime}\right|^{2}|y-x|^{2}=|x|^{2}|y|^{2}+r^{4}-2 r^{2}(x \cdot y)=\left.\left.|y|^{2}\left|x-r^{2} y /\right| y\right|^{2}\right|^{2},
$$

and thus for $|x|>r$

$$
g_{r}(x, y)=K|y-x|^{\alpha-N}\left\{1-\Phi_{r}\left(\left.\frac{y}{|y-x|}\left|x-r^{2} y /\right| y\right|^{2} \mid\right)\right\} .
$$


To compute $g_{r}(x, y)$ for $|x|<r$, note that an inversion in the sphere $S_{r}$ sends $x \rightarrow \bar{x}=r^{2} x /|x|^{2}, y \rightarrow \bar{y}=r^{2} y /|y|^{2}$ and that $|\bar{x}|>r$. Using (4.1) and some simple computations we easily obtain that

$$
g_{r}(x, y)\left(r^{2} /|x||y|\right)^{\alpha-N}=g_{r}(\bar{x}, \bar{y})
$$

Thus for $|x|<r$,

$$
\begin{aligned}
g_{r}(x, y) & =K|y-x|^{\alpha-N}\left\{1-\Phi_{r}\left((\bar{y})^{\prime}\right)\right\} \\
& =K|y-x|^{\alpha-N}\left\{1-\Phi_{r}\left(\left.\frac{\bar{y}}{|\bar{y}-\bar{x}|}\left|\bar{x}-\bar{y} r^{2} /\right| \bar{y}\right|^{2} \mid\right)\right\} \\
& =K|y-x|^{\alpha-N}\left\{1-\Phi_{r}\left(\left.\frac{y|x|}{|y-x||y|}\left|y-x r^{2} /\right| x\right|^{2} \mid\right)\right\} \\
& =K|y-x|^{\alpha-N}\left\{1-\Phi_{r}\left(\left.\frac{y}{|y-x|}\left|x-y r^{2} /\right| y\right|^{2} \mid\right)\right\}
\end{aligned}
$$

where the last equality follows from the symmetry of $g_{r}(x, y)$ and the fact that $\Phi_{r}(t)$ is a function of $|t|$. Combining the above results we obtain

THEOREM 4.1. The Green's function of the sphere is given by

$$
g_{r}(x, y)=K|y-x|^{\alpha-N}\left\{1-\Phi_{r}\left(\left.\frac{y}{|y-x|}\left|x-y r^{2} /\right| y\right|^{2} \mid\right)\right\}
$$

where $\Phi_{r}$ is the hitting probability given in (2.6).

For $\alpha=2, N>2$, the above Green's function is the classical one for the Laplacian. To see this, note that the first and second equality in 4.3 and a little computation shows that for $|x|>r$,

$$
\left[\left|y^{\prime}\right|^{2}-r^{2}\right]|y-x|^{2}=\left[|x|^{2}-r^{2}\right]\left[|y|^{2}-r^{2}\right] .
$$

Hence for $|x|>r,\left|y^{\prime}\right|>r$ iff $|y|>r$. It follows that $g_{r}(x, y)=0$ if either $|x|>r$, $|y| \leqq r$ or $|x| \leqq r,|y|>r$, while for $|x|<r,|y|<r$ or $|x|>r,|y|>r$

$$
g_{r}(x, y)=K|y-x|^{2-N}-\left.\left.K|y / r|^{2-N}\left|x-r^{2} y /\right| y\right|^{2}\right|^{2-N}
$$

For $\alpha=N=2$, i.e., . planar Brownian motion, the Green's function of the circle is just the classical Green's function for the Laplacian, and may be found in all books on partial differential equations. For $\alpha>1=N$, the Green's function of the sphere was computed in [7].

\section{BIBLIOGRAPHY}

1. R. M. Blumethal and R. Getoor, Markov processes and potential theory, Academic Press, New York, 1968.

2. - Local times for Markov processes, Z. Wahrscheinlichkeitstheorie und Verw. Gebiete 3 (1964), 50-74. 
3. R. M. Blumenthal, R. Getoor and D. B. Ray, On the distribution of first hits for symmetric stable processes, Trans. Amer. Math. Soc. 99 (1961), 540-554.

4. A. Erdelyi (Bateman Manuscript Project), Tables of integral transforms, Vol. I, McGrawHill, New York, 1954.

5. - Higher transcendental functions, Vol. I, McGraw-Hill, New York, 1953.

6. K. Ito and H. P. McKean, Jr., Diffusion processes and their sample paths, Springer-Verlag, Berlin, 1965.

7. S. C. Port, Hitting times and potential for recurrent stable processes, J. Analyse Math. 20 (1967), 371-395.

8. M. Riesz, Intégrales de Riemann-Liouville et potentiels, Acta Sci. Math. (Szeged) 9 (1938), $1-42$.

UNIVERSITY OF CALIFORNIA,

Los ANGeles, California 